\title{
A symmetric prior for multinomial probit models
}

\author{
Lane F. Burgette* and David Puelz ${ }^{\dagger}$ and P. Richard Hahn ${ }^{\ddagger}$
}

\section{Appendix}

\section{Full conditional distributions for the Gibbs sampler}

In the following sections, we provide expressions for the conditional distributions used in the Gibbs sampler. The full conditionals that are standard distributions are determined by extracting relevant components from the joint distribution of all parameters. The means and variances for the truncated univariate normal distributions follow from McCulloch and Rossi (1994).

\section{Step 1: Draw $\tilde{W} \mid Y, \tilde{\beta}_{b}, b, \Sigma_{b}, \alpha$}

To sample $\tilde{W}$, we iterate one-by-one through the elements of $\tilde{W}_{i, b}$. Note that $\tilde{w}_{i, b}$ is known given $b$ and $\tilde{W}_{i, b}$. After dropping the $b$ th element of $\tilde{W}_{i}$ and the corresponding elements in $X_{i}$ and $\beta$, the full conditionals of elements of $\tilde{W}_{i, b}$ are truncated univariate normal. The conditional means and variances can be calculated as described by McCulloch and Rossi (1994), using $\tilde{\beta}_{b}$ as the coefficient vector and $\alpha^{2} \Sigma_{b}$ as the covariance. The truncations are:

- If $Y_{i}=j \neq b$, sample $\tilde{w}_{i j}$ from a truncated normal so that $\tilde{w}_{i j}>-.5 \sum_{k \notin\{j, b\}} \tilde{w}_{i k}$ and $\tilde{w}_{i j}>\max \left(\tilde{w}_{i k}\right.$ : $k \notin\{j, b\})$.

- If $Y_{i} \neq b$ and $Y_{i}=k \neq j$, sample $w_{i j}^{\star}$ from a truncated normal so that $\tilde{w}_{i j}<\tilde{w}_{i k}$ and $\tilde{w}_{i j}>$ $-\sum_{l \neq b} \tilde{w}_{i l}-\tilde{w}_{i k}$.

- If $Y_{i}=b$, sample $\tilde{w}_{i j}$ from a truncated univariate normal such that

$$
\tilde{w}_{i j}<\min \left\{-.5 \sum_{k \notin\{b, j\}} \tilde{w}_{i k},-1\left(\max \left\{\tilde{W}_{-\{j, b\}}\right\}+\sum_{k \notin\{b, j\}} \tilde{w}_{i k}\right)\right\} .
$$

\section{Step 2: Draw $\tilde{\beta}_{b} \mid Y, b, \Sigma_{b}, \tilde{W}, \alpha$}

The transformed coefficient vector is drawn according to a normal distribution. This arises from the normal likelihood coupled with the normal prior specified for the coefficient vector, and standard Bayesian linear regression analysis specifies the conditional moments in closed form. They are as follows:

$$
\begin{aligned}
& \hat{\beta}_{b}=\left[\sum_{i=1}^{n} X_{i, b}^{\top} \Sigma_{b}^{-1} X_{i, b}+A^{-1}\right]^{-1}\left[\sum_{i=1}^{n} X_{i, b}^{\top} \Sigma_{b}^{-1} \tilde{W}_{i, b}\right], \\
& \tilde{\beta}_{b} \sim \operatorname{normal}\left(\hat{\beta}_{b}, \alpha^{2}\left(\sum_{i=1}^{n} X_{i, b}^{\top} \Sigma_{b}^{-1} X_{i, b}+A^{-1}\right)^{-1}\right) .
\end{aligned}
$$

This update follows from the fact that if $\beta_{b} \sim \operatorname{normal}(0, A)$, then $\tilde{\beta}_{b} \sim \operatorname{normal}\left(0, \alpha^{2} A\right)$.

*RAND Corporation

${ }^{\dagger}$ The University of Chicago, Booth School of Business

‡Arizona State University 


\section{Step 3: Draw $\alpha, \Sigma_{b}, b \mid Y, \tilde{\beta}_{b}, \tilde{W}$}

The draw from this distribution is divided into several steps. First, draw $b \mid \tilde{\beta}, \tilde{W}$. Second, by working with the intermediate quantity $\tilde{\Sigma}_{b}$, draw $\tilde{\Sigma}_{b} \mid b, \tilde{\beta}, \tilde{W}$. Once obtained, set $\alpha=\sqrt{\operatorname{tr}\left(\tilde{\Sigma}_{b}\right) /(p-1)}$, followed by $\Sigma_{b}=\tilde{\Sigma}_{b} / \alpha^{2}$. In this way, $\operatorname{tr}\left(\Sigma_{b}\right)=p-1$ at each iteration as in Burgette and Nordheim (2012).

Computationally, the major change from Burgette and Nordheim $(2012)$ is the draw from $\left(b, \tilde{\Sigma}_{b}\right)$. We derive this draw by first noting that from the full conditional we have:

$$
\begin{aligned}
p\left(b, \tilde{\Sigma}_{b} \mid \text { all }\right) \propto & \exp \left\{-.5 \sum\left(\tilde{W}_{i, b}-X_{i, b} \tilde{\beta}_{b}\right)^{\top} \tilde{\Sigma}_{b}^{-1}\left(\tilde{W}_{i, b}-X_{i, b} \tilde{\beta}_{b}\right)\right\} \\
& \times\left|\tilde{\Sigma}_{b}\right|^{-n / 2} p\left(\tilde{\Sigma}_{b} \mid b\right) p(b) \\
\propto & \exp \left\{-.5 \operatorname{tr}\left(\tilde{\Sigma}^{-1}\left(S_{b}+\sum\left(\tilde{W}_{i, b}-X_{i, b} \tilde{\beta}_{b}\right)\left(\tilde{W}_{i, b}-X_{i, b} \tilde{\beta}_{b}\right)^{\top}\right)\right)\right\} \\
& \times\left|\tilde{\Sigma}_{b}\right|^{-.5\left(n+\nu_{0}+p\right)}
\end{aligned}
$$

Integrating over the variance, we obtain the multinomial draw for $b$ as:

$$
\begin{aligned}
p(b \mid \tilde{\beta}, \tilde{W}) & \propto \int p\left(b, \tilde{\Sigma}_{b} \mid \text { all }\right) d \tilde{\Sigma}_{b} \\
& \propto\left|S_{b}+\sum\left(\tilde{W}_{i, b}-X_{i, b} \tilde{\beta}_{b}\right)\left(\tilde{W}_{i, b}-X_{i, b} \tilde{\beta}_{b}\right)^{\top}\right|^{-\left(n+\nu_{b}\right) / 2}
\end{aligned}
$$

Then, conditional on $b, \tilde{\Sigma}_{b}$ can be sampled from an inverse-Wishart distribution as shown in the full conditional above and described by Imai and van Dyk (2005).

\section{References}

Burgette, L. and Nordheim, E. (2012). "The trace restriction: An alternative identification strategy for the Bayesian multinomial probit model." Journal of Business and Economic Statistics, 30(3): 404-410.

Imai, K. and van Dyk, D. (2005). "A Bayesian analysis of the multinomial probit model using marginal data augmentation." Journal of Econometrics, 124(2): 311-334.

McCulloch, R. and Rossi, P. (1994). "An exact likelihood analysis of the multinomial probit model." Journal of Econometrics, 64(1): 207-240. 